\title{
Review
}

\section{Investigating cystic lung disease: a respiratory detective approach}

The cystic lung diseases are rare orphan lung disorders that most physicians will see infrequently in their everyday practice. Diagnostic and treatment options have improved over recent decades, with opportunities for slowing rate of progression and improving outcome for patients. This review provides a summary of the clinical approach to these lung disorders, including how to differentiate between different imaging patterns, clinical features, differential diagnosis and characteristics of the commonest presenting disorders.

\section{Educational aims}

- To understand the clinical, pathological and radiological features of cystic lung disorders

- To explore the differential diagnosis of cystic lung disease

- To be familiar with the key features (clinical, radiological, physiological and pathological) of the commoner cystic lung diseases, which assist in differentiating between these

\section{What is cystic lung disease?}

The term cystic lung disease includes a group of diverse pulmonary disorders, characterised by the presence of parenchymal cysts on respiratory imaging. Most of the disorders associated with multiple lung cysts (hereafter called cystic lung diseases) are rare or "orphan" lung conditions, defined as affecting fewer than one in 2000 people [1, 2]. The actual number of cysts needed to diagnose multiple cystic lung disease is usually taken to be more than five in total [3]. Population screening studies have shown that single and small numbers of cysts occur in $7.6 \%$ of normal people over the age of 40 years, and are probably a normal part of ageing. More than five cysts are very rare
@ERSpublications

Cystic lung diseases are uncommon disorders with a wide differential diagnosis. Treatment has improved over the last decade and respiratory physicians should feel encouraged to investigate such cases thoroughly to reach a final diagnosis. https://bit.ly/2W6Is9D
Cite as: Ennis S, Silverstone EJ, Yates DH. Investigating cystic lung disease: a respiratory detective approach. Breathe 2020; 16: 200041. 
Table 1 Causes of cystic lung disease

\section{Associated with genetic mutations}

Neoplastic

\section{Associated with lymphoproliferative disorders}

\section{Associated with infections}

\section{Occupational and environmental}

\section{Associated with interstitial lung disease}

\section{Conditions that may mimic cystic lung disease}

Lymphangioleiomyomatosis (tuberous sclerosis-associated and sporadic)

Birt-Hogg-Dubé syndrome and other folliculin deficiencies

Proteus syndrome, neurofibromatosis, Ehlers-Danlos syndrome, Marfan syndrome

Pulmonary Langerhans cell histiocytosis, other histiocytoses (e.g. Erdheim-Chester disease)

Metastatic sarcoma, cavitating adenocarcinomas

Benign metastasising leiomyoma

Lymphocytic interstitial pneumonia

Follicular bronchiolitis

Amyloidosis

Light chain deposition disease

$\lg _{4}$-related disease

Pneumocystis jirovecii

Staphylococcal pneumonia

Respiratory papillomatosis

Parasitic and fungal diseases (e.g. coccidiocomycosis, paragonimiasis)

Hypersensitivity pneumonitis (e.g. farmer's lung, bird fanciers' lung)

Hard metal lung disease (tungsten carbide sensitisation)

Chronic beryllium disease (beryllium sensitisation)

Respiratory bronchiolitis associated interstitial lung disease (smoking/? vaping)

Hut lung (biomass fuels)

Hypersensitivity pneumonitis

Honeycombing in idiopathic pulmonary fibrosis

Honeycombing in asbestosis and other pneumoconioses

Emphysema

$\alpha_{1}$-antitrypsin deficiency

Bronchiectasis

Sarcoidosis

Cavities in rheumatoid arthritis, Crohn's disease, coal workers' pneumoconiosis (Caplan's syndrome) in asymptomatic subjects (0.9\%) [4]. There is no globally agreed classification of cystic lung diseases and a cause-based classification is usually used (table 1); some excellent reviews of this topic are available [1, 2, 5-7]. Because of the complexity of this area and the need for specialised investigations, coming to a final diagnosis may take some time; however, using a "respiratory detective" approach can usually allow a definite diagnosis, which helps greatly with management and treatment.

Most respiratory physicians will not see more than one case of cystic lung disease a year. This means that individual experience may be limited. Patients (and physicians) may feel "orphaned", or lonely and isolated about the case. Patients may request referral to specialised academic clinics and increasingly find their way to experts via the internet. A lot of information is available from "Dr Google" for patients about their possible diagnosis, and not all of this is reliable. Thus, patients can be very anxious about these potentially fatal diseases.

Over the last two decades, aided by vigorous patient organisations and improved research funding, there have been great improvements in this field which have led to targeted treatments and the possibility of much improved outcomes. This has been accompanied by excellent guidelines for the investigation and treatment of individual disorders [9-11], allowing the generalist some reassurance about individual patient management. Implementation of regular webinars, podcasts and international clinical collaborations among respiratory and other clinicians has also greatly assisted in finding the best possible treatments, albeit sometimes limited by practical resources.

Knowledge about how to classify these disorders is rapidly evolving. As always with classification 
systems, categorising different cystic lung diseases is imperfect. Causes can be thought of as falling into four main groups:

- those associated with defined genetic mutations

- neoplasms including the lymphoproliferative disorders

- cystic lung change associated with the commoner interstitial lung disorders ("autoimmune" and immunological disorders)

- due to external agents (occupational and environmental exposures, and particular infections)

All these may of course occur on the background of a pre-existing respiratory or genetic disorder. Exploring these diseases makes one aware of the inadequacies of our current paradigms regarding disease classification, diagnostic test accuracy, the function of particular cells and the actual nature of malignancy.

This paper attempts to provide a clinical approach to the diagnosis of cystic lung diseases. It is limited to a brief outline of individual disorders, and readers are referred to the excellent detailed reviews and guidelines for further information [1-11].

\section{What is a cyst?}

When first assessing a patient with cystic lung disease, it is important to decide whether they actually have this.

A cyst is defined as "any round circumscribed space that is surrounded by an epithelial or fibrous wall of various thickness" [3]. Cysts have thin walls $(<2 \mathrm{~mm})$ and are not usually associated with pulmonary emphysema. The surrounding lung is usually normal. On high-resolution computed tomography (HRCT), cysts appear as a round, parenchymal lucency (low-attenuating area) that can easily be distinguished from the surrounding lung. Cysts in the lung usually contain air, but occasionally can be filled with fluid or solid material [3]. Some can start as solid lesions and become thin walled once any acute pathology has resolved $[1,3,11]$.

It is important to distinguish true cysts from other lung abnormalities including emphysema, bullae, lung cavities and honeycombing. The main difference between a cyst and a bulla is its size. Bullae are large regions of lucency within the lung, also with thin walls $(<1 \mathrm{~mm})$, but are $>0.5 \mathrm{~cm}$ in diameter, and sometimes large, being bordered only by remnants of alveolar septae and/or pleura [3]. Bullae have been described after environmental exposures such as tobacco smoking, marijuana smoking, genetic disorders and HIV infection. The possibility of $\alpha_{1}$ antitrypsin deficiency should always be borne in mind with any bullous, cystic or emphysematous lung disease. A "bleb" was the term formerly used for a subpleural bulla, often when associated with emphysema, but this word has now gone out of fashion. Areas of emphysema can be usually be distinguished from cysts because they do not have any walls and the central dot (representing the pulmonary artery) can often also be seen. However, the distinction between cysts and emphysema can sometimes be very difficult, especially when cysts coalesce to produce large areas [1, 3, 4].

Pneumatoceles are another lucent area in the lung that can cause confusion. These are a type of thin-walled parenchymal cyst with an epithelial or fibrous lining. They arise from overdistension of the lung after identifiable events such as barotrauma from mechanical ventilation, acute bacterial or Pneumocystis jirovecii pneumonia, or chest trauma. They often (but not always) resolve after treatment of the underlying cause [4, 5].

Cysts are generally easy to distinguish from cavities, which are thick walled ( $>4 \mathrm{~mm}$ ) and usually accompany an area of consolidation, mass or nodule. Their contents can include debris or fluid in addition to air. Localised areas of lucency occurring with interstitial pulmonary fibrosis are also not, strictly speaking, cysts. Honeycombing, which is often found in idiopathic pulmonary fibrosis and asbestosis, occurs as a result of interstitial pulmonary fibrosis causing contraction of the lung parenchyma, producing enlarged airspaces with thick fibrous walls and architectural distortion. Honeycombing usually occurs subpleurally and may be accompanied by traction bronchiectasis. Cystic bronchiectasis looks different from lung cysts, with thick walled, nontapering bronchi producing "tram tracks", ring opacities and tubular structures representing filled airways.

In all cystic lung diseases, careful examination of the neighbouring lung parenchyma can be very helpful in attempting to discern the cause.

The pathogenesis of pulmonary cyst formation and bullae is not well understood. Several mechanisms have been proposed, but none adequately verified [1, 2]. It seems likely that the pathogenesis differs according to the underlying aetiology. One suggested mechanism is of a bronchiolar check-valve, with obstruction followed by distal overinflation. The entrance from the bronchus into the lung becomes obstructed in a valve-like manner, allowing air entry but not exit. This is proposed to result in balloonlike expansion of distal airways. Other proposed mechanisms include ischaemia, direct infiltration and lung parenchymal remodelling induced by metalloproteinases and other matrix-degrading enzymes [1, 2, 6]. It seems likely that in most cases, more than one mechanism is involved.

\section{Suspecting and making a diagnosis of cystic lung diseases}

\section{Clinical history}

Pneumothorax use to be the most common acute presentation of the multiple cystic lung 
diseases, particularly lymphangioleiomyomatosis (LAM) [6-10], but patients are increasingly being diagnosed after incidental detection of lung cysts on routine imaging of the lungs or other parts of the body (e.g. abdominal CT, when cuts of the lower lungs are routinely included). A lung cyst must be very large to be detectable on a chest radiograph; nowadays, the patient has usually had a CT scan. Pneumothorax is the traditional acute presentation and should be regarded as a sentinel event, particularly in a young woman [7]. Despite the role of tobacco, younger patients have rarely had enough time to smoke enough for this be the reason for a pneumothorax, so other causes should always be considered. Recurrent pneumothorax should always alert the clinician to the possibility of underlying lung disease. However, many patients will present with nonspecific symptoms such as cough, breathlessness or chest pain, and to other specialties.

It is important to take a careful history of previous pneumothoraces and other lung exposures such as tobacco smoking, vaping or drug use, although the fact that someone has smoked should not make one dismiss the possibility of another cause. Taking a thorough occupational, family and past medical history including previous skin and kidney disorders is required. Information about past haematological problems or malignancy can be diagnostic.

A family history can take time but is helpful, and sometimes can clinch a diagnosis (e.g. in folliculin deficiencies). This should ideally extend back for three generations and be documented by drawing a complete family history. Often, patients will not recognise the term "pneumothorax" and therefore, using the term "collapsed lung" may be needed. If the clinician suspects a diagnosis of, for example, tuberous sclerosis complex (TSC), then inquiring about a family history of epilepsy, renal disease or learning difficulties is useful. The absence of any family history does not, however, exclude disease, as new mutations are relatively common.

\section{Examination}

Careful examination of the patient can be diagnostic. Specific skin lesions are associated with several causes of cystic lung disease (table 2) and signs of autoimmune diseases may also be found. Remember to examine as much of the skin surface as is possible, including the legs and feet, armpits, scalp, and mouth. The skin signs of TSC, an autosomal dominant hamartomatous syndrome associated with LAM, can be subtle and occasionally the only abnormality will be a single hypopigmented patch. Subungual fibromas in TSC and nail pitting are easily missed. Nailfold capillaroscopy may be helpful, as well as skeletal and joint examination.

Although skin lesions in genetic disorders may be impressive, their absence does not exclude disease. Patients nowadays are increasingly taking advantage of cosmetic procedures as well as screening tests and may have had a relevant skin abnormality removed. In addition, there is a lot of variability in skin manifestations, which tend to progress with age, so these might yet not yet be visible. Images of skin manifestations of various genetic disorders are available online and are helpful as reminders, when such lesions are not seen frequently.

Chest auscultation is rarely diagnostic and is usually normal. Most cystic lung diseases are not associated with any added lung sounds, although interstitial pulmonary fibrosis may show fine late inspiratory crackles. Wheeze may accompany moderate or severe airflow obstruction. If a chylothorax is present in association with LAM, however, this can usually be detected.

Abdominal examination can occasionally reveal renal masses, although usually only with gross disease, and occasionally ascites can be found (with chyloperitoneum in LAM). Other system examination is usually normal but blood pressure needs to be measured (renal angiomyolipomas (AMLs) may be associated with hypertension); neurological examination may be needed in TSC.

\section{Investigations}

Useful blood tests include a full blood count and biochemical profile including liver function tests (including lactate dehydrogenase), C-reactive protein, a full autoantibody screen and urinalysis for microscopic haematuria. If an immunological disorder is suspected, immunoglobulins (including IgG subclasses) protein electrophoresis and immunoelectrophoresis may be useful as well as urinary Bence Jones protein. $\alpha_{1}$-antitrypsin testing should be performed if results are not already available. HIV or testing for other infectious agents may also be needed. Vascular endothelial growth factor (VEGF)-D testing in LAM can be helpful diagnostically [10] but is not available in most centres. Further blood and other investigations including bone marrow examination may be ordered later, depending on initial results.

Full lung function testing is useful at baseline, particularly to assess total lung capacity, diffusing capacity of the lung for carbon monoxide $\left(D_{\mathrm{LCO}}\right)$ and arterial blood gases, but is nonspecific. The presence of normal spirometry by no means excludes disease. Lung function is best performed, where possible, using body plethysmography, as lung volumes may be raised in association with gas trapping. An obstructive deficit is most common, surprisingly sometimes with a significant bronchodilator response [9, 10], which can lead to a mistaken diagnosis of asthma. Mixed deficits can accompany previous pneumothorax with pleurodesis or lung parenchymal involvement (e.g. in autoimmune or interstitial diseases). Serial lung function testing is extremely useful for monitoring progression, particularly in monitoring $D_{\text {Lco }}$ changes, and 6-min walk distance can also 


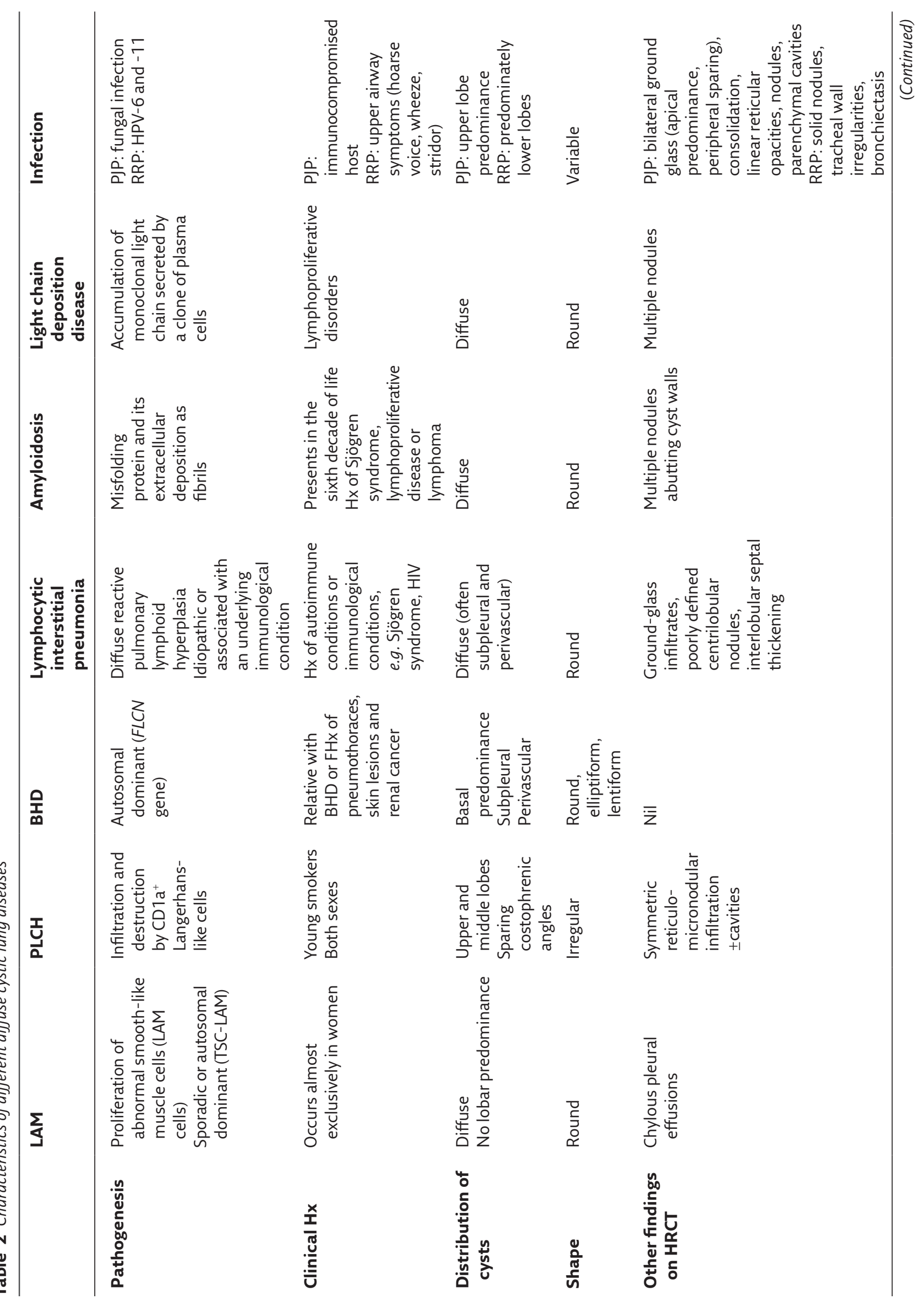




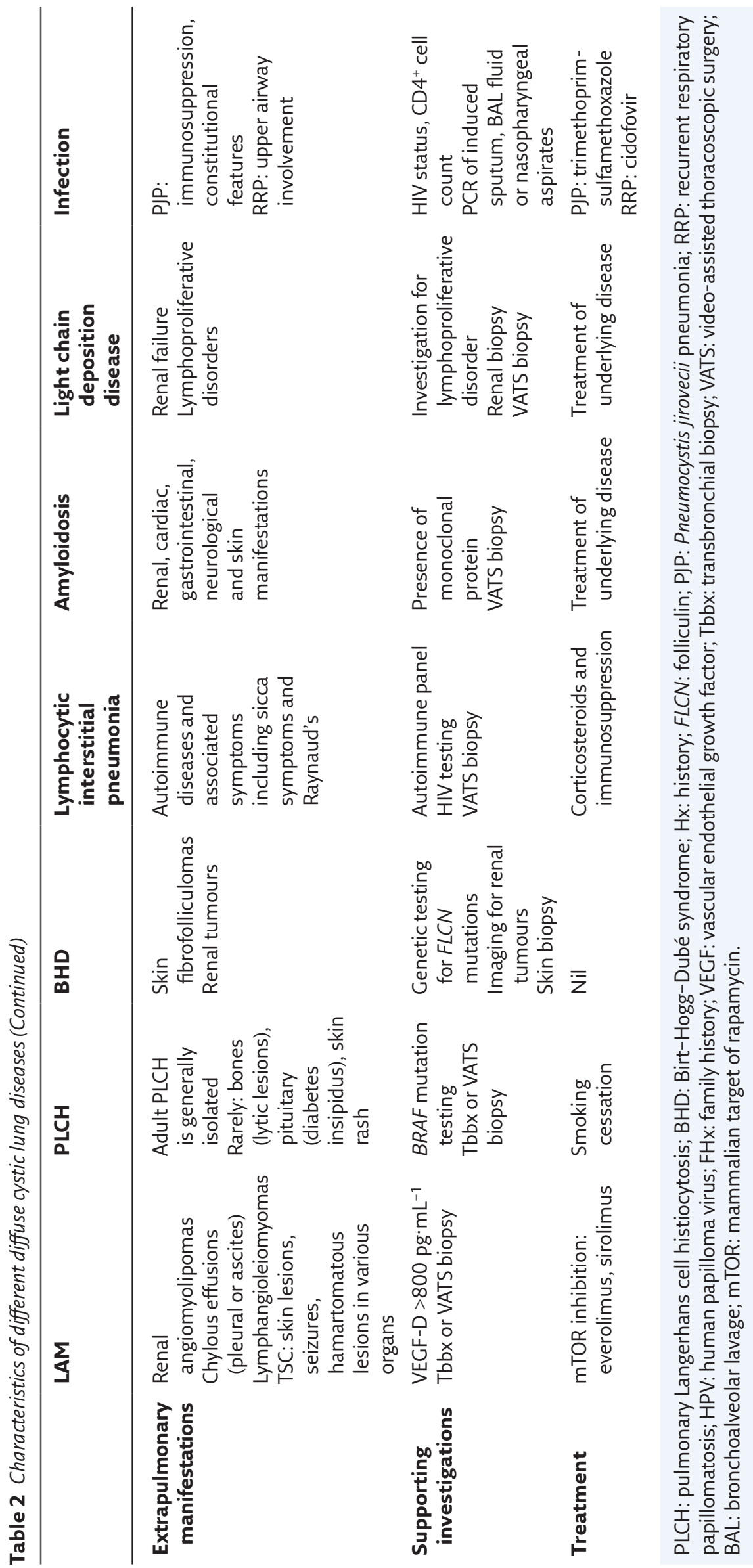


be used. Normal predicted longitudinal values for lung function loss are available for LAM [10] and for occupational disorders (SPIROLA software at www.NIOSH.com). Cardiopulmonary exercise testing may pick up early disease but this is not usually necessary, as most patients usually have demonstrably abnormal lung function by the time they are referred.

The increasing availability and quality of $\mathrm{CT}$ has assisted greatly in the diagnosis and assessment of cystic lung disease [3, 4, 7], and has been shown to be cost-effective in screening after pneumothorax. The size, distribution and presence of associated lung pathology are very useful in narrowing the differential diagnosis, and automated methods are now available for accurate assessment of the total volume occupied by the lung cysts. Comparison of serial imaging is extremely valuable in assessing disease and rate of progression. Imaging of other areas (e.g. the abdomen and pelvis in LAM, the head in TSC) may detect abnormalities (e.g. renal) and thus allow diagnostic certainty. It can be difficult in clinical practice to differentiate between emphysema and multiple pulmonary cysts, especially if a patient has smoked, although this uncertainty is not reflected in the radiological literature. A thoracic radiologist's opinion can be invaluable in such cases, and examination of serial imaging is frequently helpful. The main differentiating characteristics on $\mathrm{CT}$ are shown in box 1.

A clinical diagnosis is not made based on a CT alone, and a thorough clinical history, examination and HRCT will reveal further clues about a possible diagnosis. Further imaging may reveal diagnostic lesions (e.g. renal lesions) or a skin biopsy will come back revealing a fibrofolliculoma (e.g. with Birt-Hogg-Dubé syndrome (BHD)). The aim is always to make the diagnosis using the least invasive tests.

Genetic testing is invaluable, in particular, testing for TSC1 and TSC2 mutations in cases of suspected TSC or LAM, and for folliculin (FLCN) mutations, after referral to a geneticist. Testing can be performed on blood, skin or other tissue. Targeted testing may be used but the availability of whole-genome sequencing allows for potential identification of new mutations if applicable. Genetic testing is resourcelimited and may take several months to come through; patients often do not understand the need for genetic counselling, which is mandatory, and the need for referral to another doctor. Thus, it is helpful to explain this carefully to them, including the fact that they may find the whole process somewhat frustrating, and that it is very worthwhile, particularly if it avoids more invasive tests.

Respiratory investigations are, on the whole, not usually diagnostic. Pleural aspiration and fluid analysis can diagnose a chylothorax but usually does not reveal the cause, as most laboratories are not able to identify LAM cells in pleural fluid. Bronchoscopy with transbronchial biopsy can be attempted, but sufficient tissue for a diagnosis is not always obtained and there is a higher risk of pneumothorax than with other conditions. Positron emission tomography-CT can be used in cases of potential malignancy and lymphoproliferative disorders, but is also nondiagnostic.

Most of these disorders will ultimately require a surgical lung biopsy, often using a videoassisted approach, to collect enough tissue to make a definitive diagnosis. This is invasive, but often worthwhile in the long run, as specific management can usually be implemented. Lung biopsy is the gold standard for the diagnosis of sporadic LAM, Langerhans cell histiocytosis, amyloidosis and most other disorders. Because LAM is a diffuse infiltrative disease, sampling problems are unusual. One does, however, need to alert the pathologist to the possibility of LAM, as HMB-45 staining for LAM cells is not routinely performed. BHD is an example of a pathology that will not be obvious on histopathology, where nonspecific cysts are found.

Even with full investigation, however, sometimes the disease is unclassifiable (cryptic cause). Such cases need to be put in the "cold case" basket; they are probably unrecognised new disorders that we will come to learn about in the future. Even a negative finding can, however, be helpful for the patient if it avoids long-term treatment and clarifies the situation. The advantage of having a surgical biopsy is that tissue can be re-examined and stored for the future, when using new techniques or diagnoses may be applicable.

Careful collation of all the available information about a patient is essential. Patients may have forgotten about previous medical issues or the relevance may not have been appreciated. Any patient with multiple pneumothoraces should be further investigated. The advent of electronic medical records should have assisted with collation of results but cannot currently be fully relied upon, especially for medical issues long ago. Thus, searching earlier records may be needed. Finding a history of a previous renal AML can, for example, be diagnostic and save a patient from unnecessary investigations [8].

Once all information is available, it is usually possible to decide on a likely diagnosis and arrange further treatment. Consultation with other colleagues is very helpful, particularly with geneticists, who can order appropriate gene testing, as well as dermatologists, pathologists, immunologists, haematologists and surgeons.

With such rare disorders, expert pathological and management opinions are often needed, and these have been facilitated by international collaborative groups that include physicians, radiologists and pathologists who are interested enough to kindly give second opinions. The US LAM Foundation, for example, organises regular conference calls for all its designated LAM clinicians, so that individual cases can be discussed. 
Box 1. Differential diagnosis of lung cysts: bulla, emphysema, pneumatocele, honeycomb lung

\begin{tabular}{|c|c|}
\hline \multirow{2}{*}{$\begin{array}{r}\text { Centrilobular } \\
\text { emphysema }\end{array}$} & Centrilobular lucencies that lack distinct walls \\
\hline & Predominately upper lobes after tobacco smoking \\
\hline \multirow{2}{*}{$\begin{array}{l}\text { Cystic } \\
\text { bronchiectasis }\end{array}$} & Associated with bronchial dilation \\
\hline & Connected to tubular airways \\
\hline \multirow[t]{3}{*}{ Cavity } & Gas-filled space \\
\hline & Wall thickness $>4 \mathrm{~mm}$ \\
\hline & Develops in an area of pulmonary consolidation, mass or nodule \\
\hline Bulla & An airspace (size $>1 \mathrm{~cm}$ ) with a round focal lucency, bounded by a thin wall $(<1 \mathrm{~mm})$ \\
\hline Bleb & Small sized $(<1 \mathrm{~cm})$, gas-containing space within the visceral pleura or in the subpleural area \\
\hline Pneumatocele & A thin-walled, gas-filled space in the lung, caused by infection, trauma, aspiration of hydrocarbon fluid \\
\hline Honeycombing & Subpleural, multiple cystic cluster, typically 3-10 mm in diameter with well-defined walls \\
\hline
\end{tabular}

\section{Most likely causes of multiple cystic lung diseases}

There are many interesting causes of cystic lung disease but it is not possible to discuss all of them in this article. Table 1 lists the main causes and a brief summary of the most important facts regarding individual diseases is shown in table 2 . The most likely diseases causing cystic change disease are LAM, pulmonary Langerhans cell histiocytosis $(\mathrm{PLCH})$ and $\mathrm{BHD}$, which are described briefly below, along with a short summary of some other causes.

\section{Cystic lung diseases associated with genetic mutations}

Lymphangioleiomyomatosis

LAM is as a multisystem genetic disease resulting from mutations in the tumour suppressor genes TSC1 and TSC2. Abnormal tumour-like cells (LAM cells) infiltrate the lung parenchyma
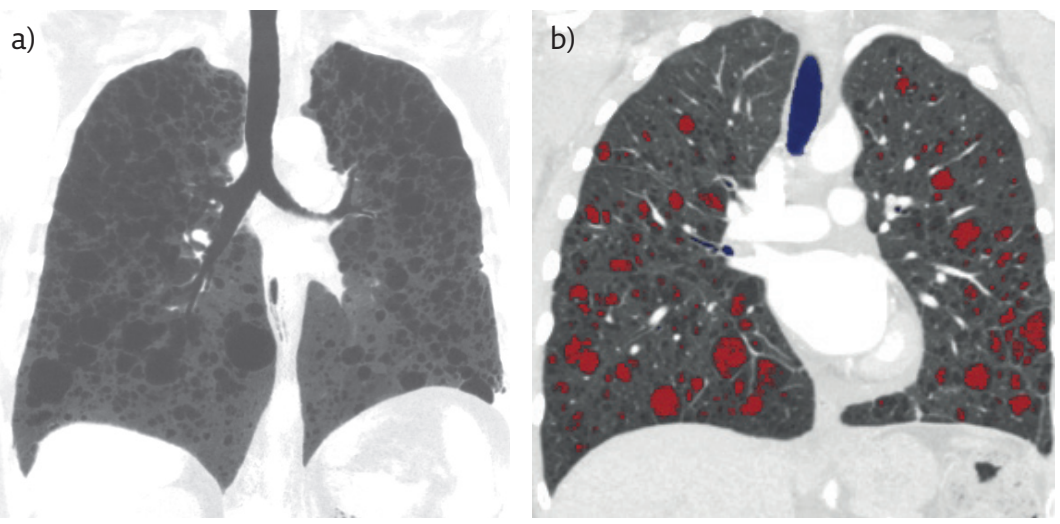

Figure 1 a) HRCT image of the lungs in sporadic LAM, demonstrating bilateral rounded cysts, and $b$ ) automated CT software image.
[7-10]. Multiple small thin-walled cysts result, evenly distributed within both lungs (figure 1). Extrapulmonary manifestations include benign tumours called renal AMLs and abdominal masses called lymphangioleiomyolipomata. These are cystic retroperitoneal tumours of the abdomen, retroperitoneum or pelvis containing lymphatic, blood, smooth muscle and fatty tissue. LAM causes chylothorax and pneumothorax, and almost exclusively affects women of reproductive age. LAM can be either sporadic or associated with TSC.

Excellent guidelines are available for diagnosis and treatment [8-10]. LAM is classified initially into possible, probable or definite LAM. Definite LAM is diagnosed if the patient has a compatible history and characteristic HRCT of the chest and one or more of the following features: TSC, renal AML, elevated VEGF-D ( $\left.\geq 800 \mathrm{pg} \cdot \mathrm{mL}^{-1}\right)$, chylous effusion (pleural or ascites), lymphangioleiomyomas, demonstration of LAM cells or LAM cell clusters on cytological examination of effusions or lymph nodes and histological confirmation of LAM by lung biopsy [8, 9]. For patients who have cystic changes on HRCT but no additional confirmatory features of LAM, HRCT in isolation should not be used to make a clinical diagnosis of LAM.

LAM has a variable clinical course. Older age at diagnosis and higher baseline forced expiratory volume in $1 \mathrm{~s} / D_{\mathrm{LCO}}$ at diagnosis are associated with a better prognosis [8-10], but a significant bronchodilator response to salbutamol indicates a worse prognosis [9].Treatment with mammalian target of rapamycin (mTOR) inhibitors (including sirolimus and everolimus) has revolutionised the treatment of LAM and TSC. These stabilise lung function, reduce the size of AMLs and control lymphatic complications [12, 13]. They are, however, expensive and treatment is not curative. Much research effort is being directed towards combined or new treatments. 
BHD and folliculin deficiencies

BHD is a tumour suppressor gene syndrome associated with fibrofolliculomas, cystic lung disease and chromophobe renal cell carcinomas. First described in 1977 by Canadian dermatologists, it is caused by a germline mutation in the FLCN gene encoding the protein folliculin [1, 14]. Folliculin is expressed strongly in skin, distal nephrons and type I pneumocytes, and has effects on cell-cell adhesion, but its functions have not yet been elucidated. As an autosomal dominant condition, BHD affects males and females equally. FLCN mutations cause dysregulation in mTOR signalling; however, the exact pathophysiology behind the development of pulmonary cysts remains unclear $[6,14,15]$.

The most common presentation of BHD is with recurrent pneumothoraces, and there is often a family history of such and/or renal cancers [14, 15]. Female patients may be misdiagnosed as having LAM. The incidence of pneumothorax is 32 -fold higher than the general population, with an $\sim 75 \%$ likelihood of recurrence [14-16].

Compared to LAM, cysts are different in shape and distribution [15]. In BHD, they are basally predominant, classically occurring below the level of the carina [6], and also found along the mediastinum, abutting the pleura and proximal vessels (figure 2 ). They are rounded to lentiform in shape, thin walled and of variable size. Lung and skin lesions typically manifest in the third or fourth decade of life, but pneumothorax can occur in children. Lung function testing is usually within normal limits but may show obstruction with reduced $D_{\text {LCO }}$ [14-16].

Typical skin manifestations include facial papules (fibrofolliculomas and trichodiscomas), which can look like whiteheads or just unpigmented moles [1, 15]. Skin tags (acrochordae) on the head, neck and upper trunk can also occur. Renal manifestations range from benign cysts to malignant tumours. There is a seven-fold increased risk of renal cancer in BHD [16] and a questionable increased risk of colorectal cancer as well. A spontaneous pneumothorax in a young patient, particularly with a family history of pneumothoraces, skin lesions and/or renal tumours should prompt investigation for BHD [17].

Chest HRCT alone does not establish a diagnosis [15], which is made by detection of a mutation in the FLCN gene and compatible clinical features. Genetic testing necessitates referral to a geneticist and genetic counselling; patients' families should also be screened if tests are positive.

Although there are few published data, the rate of loss of lung function in BHD is slow compared, for example, with LAM. Provided the patient does not smoke, the main respiratory risk for the patient is pneumothorax, and lung disease does not usually progress to respiratory failure [15].

Early surgical pleurodesis is recommended after a first pneumothorax due to the high recurrence rate in BHD [17]. Patients should probably also avoid
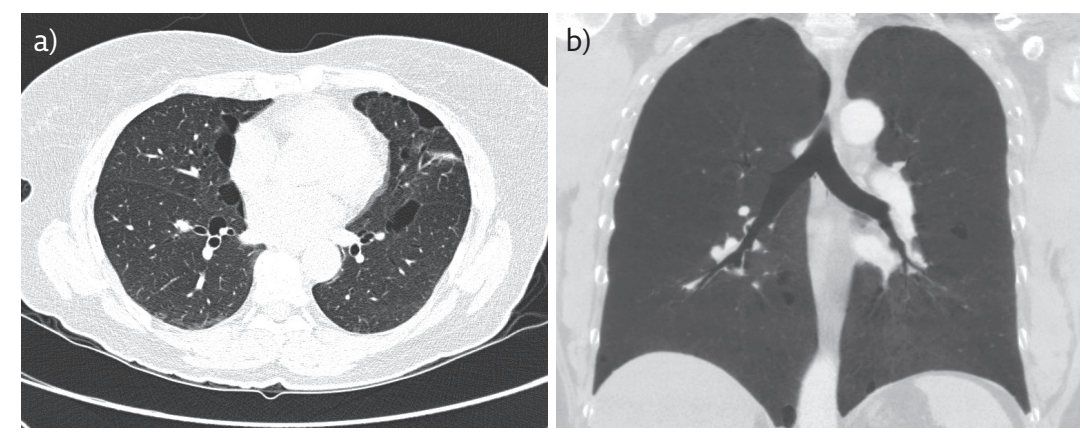

Figure 2 Typical HRCT appearances of the lungs in BHD.

diving and travel to high altitude. In practice, there is insufficient information about these risks, and it is not possible to predict when another pneumothorax will occur. This can be a source of anxiety for young, active patients who wish travel to remote regions; some opt for prophylactic pleurodesis in the absence of any good evidence on this issue. The interaction between cigarette smoking and BHD is poorly documented [16], but avoidance of smoking is sensible. No specific treatment for is available. mTOR inhibition, in theory, could be helpful but does not assist with skin lesions, and is not currently recommended. The prognosis depends primarily upon the risk of renal cancer and yearly surveillance for renal tumours is recommended [15].

\section{Neoplasms causing cystic lung disease}

\section{Pulmonary Langerhans cell histiocytosis}

$\mathrm{PLCH}$, known as histiocytosis X until 1987, is a rare systemic disorder characterised by infiltration of Langerhans cells into the lungs. After long debate about whether LCH was an immune disorder or a neoplasm, LCH has been confirmed as an inflammatory, clonally expanding myeloid neoplasm [18]. LCH can vary from a single-organ disease with spontaneous remission to a systemic and aggressive disease that can involve any organ, and can be fatal. In adults, the disease is typically isolated to the lung alone, and is not usually part of the multisystem condition affecting bone, skin, lymph nodes, pituitary and central nervous system in children [1, 18-20].

$\mathrm{PLCH}$ is a specific subtype of $\mathrm{LCH}$ and is strongly related to smoking. Distal bronchioles are infiltrated and destroyed by Langerhans cells (staining for CD1a and langerin), producing inflammatory nodules and granulomas [20]. The majority of cell proliferation in $\mathrm{PLCH}$ is polyclonal and the prognosis is generally better than with other organ involvement [21].

$\mathrm{PLCH}$ is typically seen in young adults, with no sex predilection [18-21]. Environmental exposures are believed to be involved in pathogenesis but information about this is scanty. Patients may present with pneumothorax (15-30\% of cases) or nonspecific respiratory symptoms. Diagnostic 
and treatment algorithms for $\mathrm{PLCH}$ are available [11, 18-20]. CT imaging of the abdomen and pelvis excludes hepatosplenomegaly and lymphadenopathy, and bone marrow and/or mutation testing may be required. Modern guidelines classify LCH as single-system LCH, with multifocal or unifocal involvement, or as multisystem $\mathrm{LCH}$, with multiple organ involvement with or without risk organ involvement [18]. HRCT of the chest typically demonstrates bilateral micro- and macronodules with or without cavitation, evolving into thin- and thickwalled cysts seen predominately in the upper and middle lung fields, with sparing of the costophrenic angles (figure 3) [1, 11, 18-23]. Cysts seen in PLCH are variable in size, ranging from $2 \mathrm{~mm}$ to $>2 \mathrm{~cm}$, and characteristically "bizarre" and irregular in shape.

A definitive diagnosis of $\mathrm{LCH}$ requires identification of Langerhans cell granulomas, usually after surgical lung biopsy [18]. Smoking cessation is essential and can be curative. Chemotherapy is considered for symptomatic patients with progressive disease [20] and oral corticosteroids are used in this context only. The identification of somatic mutations in the mitogen-activated protein kinase pathway, including BRAF ${ }^{\mathrm{V} 600 \mathrm{E}}$ and MAPK $2 K 1$, has led to targeted therapy with agents such as dabrafenib and vemurafenib [22]. To date, however, there are few clinical trial data.

Cystic lung diseases associated with lymphoproliferative disorders

\section{Amyloidosis}

Pulmonary amyloidosis is a rare disorder caused by deposition of amyloid misfolding proteins and extracellular fibril deposition, most often found in association with haematological abnormalities such as non-Hodgkin lymphoma, Waldenström macroglobulinaemia and mucosa-associated lymphoid tissue lymphoma [24]. Long-term chronic inflammatory conditions can also produce amyloid.

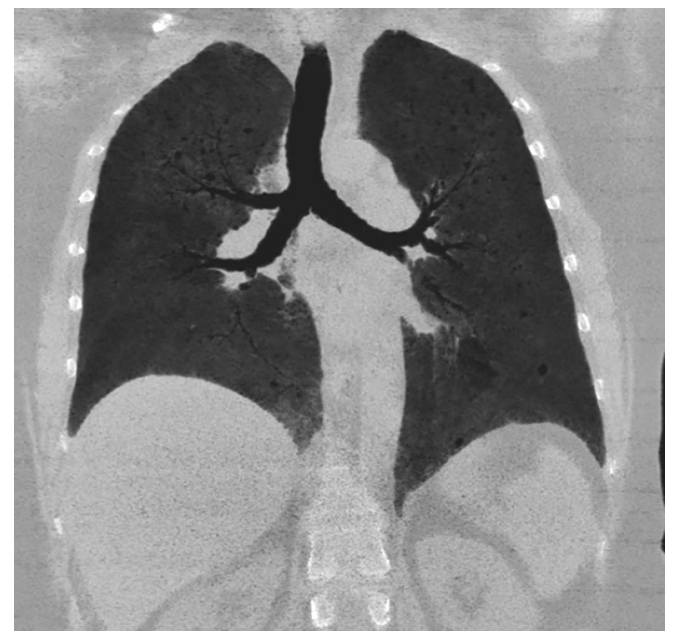

Figure 3 Lung cysts and mid and upper lobe infiltrates in biopsy-proven hypersensitivity pneumonitis, with accompanying fibrotic change. The sensitising agent was the pet parrot.
Clinically, amyloidosis usually presents in the sixth decade of life, associated with dyspnoea, cough, wheezing, haemoptysis and "recurrent pneumonia" $[1,23,24]$. On HRCT, lung cysts are small to moderate sized, thin walled, and round or lobulated in shape. They are located along the periphery of the lung parenchyma with no apicobasal gradient [1]. Small nodules may accompany cysts. Lung biopsy reveals fibrillar deposits that stain positively with Congo red. Precise diagnosis of the type of amyloidosis is important because this affects treatment. Effective chemotherapy treatment regimens have been developed, particularly for AL amyloid.

\section{Light chain deposition disease}

Light chain deposition disease (LCDD) is also a systemic disease, characterised by accumulation of immunoglobulin light chains in the lung. Light chains are secreted by a clone of plasma cells and the majority (75\%) of patients have an underlying plasma cell dyscrasia (e.g. multiple myeloma, Waldenström macroglobulinaemia or B-cell lymphoma). The distinction between amyloidosis and LCDD relies on the detection of tissue light chain fragments that do not stain with Congo red. Lung involvement in LCDD is very rare and cysts do not usually occur in the absence of nodules. Cysts are generally thin walled, spherical and diffusely distributed throughout both lungs [1, 5], but can progressively increase in number and size and coalesce [1] to produce eventual respiratory failure [25]. To confirm a diagnosis of LCDD, an open-lung biopsy is required. Treatment is that of the underlying haematological disease [25].

Other neoplastic causes: sarcoma, adenocarcinoma, benign metastasising leiomyoma and other cancers

Several types of cancer can cause lung cysts. Lesions can occur with metastatic sarcoma, where spontaneous pneumothorax may be the presenting clinical sign [1], or adeno- and squamous carcinoma [26]. Benign metastasising leiomyoma is a very rare condition affecting women of reproductive age where a benign leiomyoma of the uterus spreads to lung and bone; in contrast to LAM, it is HMB-45 negative on biopsy [27].

\section{Cystic lung disease associated with interstitial lung disorders}

Cystic lung disease can occur in association with many interstitial lung disorders, particularly Sjögren syndrome, hypersensitivity pneumonitis (HP) and sarcoidosis. Despite changes in the classification of the interstitial lung disorders recently, this should not worry the respiratory detective, as most cases are now discussed in multidisciplinary meetings; essentially, treatment is of the underlying cause. 
Lymphocytic interstitial pneumonia (LIP) is a clinicopathological term that is often associated with cystic lung disease, where diffuse involvement of lung parenchyma by reactive pulmonary lymphoid tissue occurs, often with follicular bronchiolitis [28]. Cases of LIP are now generally classified as nonspecific interstitial pneumonia or collagen vascular diffuse lung disease. LIP was a nonspecific term and associated with a wide range of disorders involving the immune system, often producing ground-glass opacities in the lower lobes. In addition to being associated with autoimmune conditions such as Sjögren syndrome, systemic lupus erythematosus, Hashimoto thyroiditis and rheumatoid arthritis, it also occurred in immunodeficiency states such as HIV and common variable immune deficiency, and after infections such as Legionella, Mycoplasma or Epstein-Barr virus. In 20\%, no cause could be identified [28]. Its importance lies in its potential for treatment.

Sarcoidosis is one of the commoner interstitial lung diseases (ILDs) and is, of course, a multisystem disorder. Lung involvement occurs in $\sim 80 \%$ of patients. Cysts are rare but well documented. Both large cysts (in severe disease) and smaller, thinwalled cysts have been described [1, 3].

$\mathrm{HP}$ is another well documented cause of lung cysts, usually in chronic rather than acute disease. Middle- and upper-lobe fibrotic change develops, with small cysts, usually only after many years of inhaling the causative allergens (figure 3) [1, 2]. Chronic HP is underdiagnosed, yet forms a significant proportion of cases presenting to ILD clinics. A thorough occupational and exposure history can be very worthwhile because early removal from exposure can prevent progression.

\section{$\operatorname{lgG}_{4}$-related disease}

$\lg _{4}$-related disease ( $\operatorname{lgG}_{4}-\mathrm{RD}$ ) is an emerging disorder first reported in 2001 [29]. It is an immune-mediated fibroinflammatory condition usually affecting the pancreas, kidneys, salivary glands and thyroid. Lung involvement is increasingly being recognised (called $\operatorname{lgG}_{4}$-related respiratory disease (lgG $\left.-R R D\right)$ ). $\operatorname{lgG}_{4}-\mathrm{RD}$ has had a variety of previous labels, including Mikulicz syndrome, sclerosing pancreatitis, inflammatory pseudotumour and retroperitoneal fibrosis, and has only recently been recognised as a single entity, with diagnostic criteria published [30]. $\operatorname{lgG}_{4}-\mathrm{RRD}$ is a great mimic and can present as almost any lung pathology. Because it responds to glucocorticoids and antirheumatic drugs, it is important to try to identify this early. Median age at diagnosis is 60 years, more often in men than women, and symptoms of asthma and/or allergy occur ( $40 \%$ of patients). Serum $\operatorname{lgG}_{4}$ levels are elevated in most (about twothirds) of patients but not all, and a tissue diagnosis is often necessary to make a diagnosis [29, 30]. Lung histopathology shows diffuse lymphoplasmacytic infiltration, obliterative vascular changes and fibrosis with occasional eosinophilic infiltration. As awareness of $\operatorname{lgG}_{4}$-RRD increases, the spectrum of airway disorders associated with $\lg _{4}-\mathrm{RD}$ is likely to expand.

\section{Occupational and environmental causes of cystic lung disease}

Probably the commonest cause of lung disease worldwide is cigarette smoking, which can produce a desquamative interstitial pneumonia, the precursor to respiratory bronchiolitis-associated ILD. This can be associated with multiple lung cysts [1, 2] but ground-glass opacification is more usually found. Vaping is a new potential cause. A careful occupational and environmental history may be time consuming but may well have important implications in cystic lung disease.

Cysts are well described with HP, an allergic reaction usually (but not always) to animal protein, resulting in a bronchiolocentric granulomatous lymphocytic alveolitis. Exposures relevant to chronic $\mathrm{HP}$ can easily be overlooked but are important, as HP is potentially curable by removal from the antigen. One differentiating feature is the presence of ground-glass opacities and/or interstitial fibrosis, usually occurring in the mid- and upper zones, and of course a history of exposure.

Several other types of occupational exposures can cause multiple lung cysts, including hard metal lung disease (HMLD) [31] and chronic beryllium disease. HMLD occurs after occupational sensitisation to tungsten carbide, and may be associated with large cystic lesions and relatively sparse fibrosis [31]. Chronic beryllium disease can look identical to sarcoidosis but has a history of exposure (often in the defence and aerospace industries). Hut lung (or domestically acquired particulate lung disease) is another interesting disorder that occurs in women exposed to dusts and fumes from cooking or burning dung or other fuels [32]. Fire eater's lung is another disease that has been associated with inhaling petroleum derivatives [1] and no doubt more diseases will be added to this list in the future.

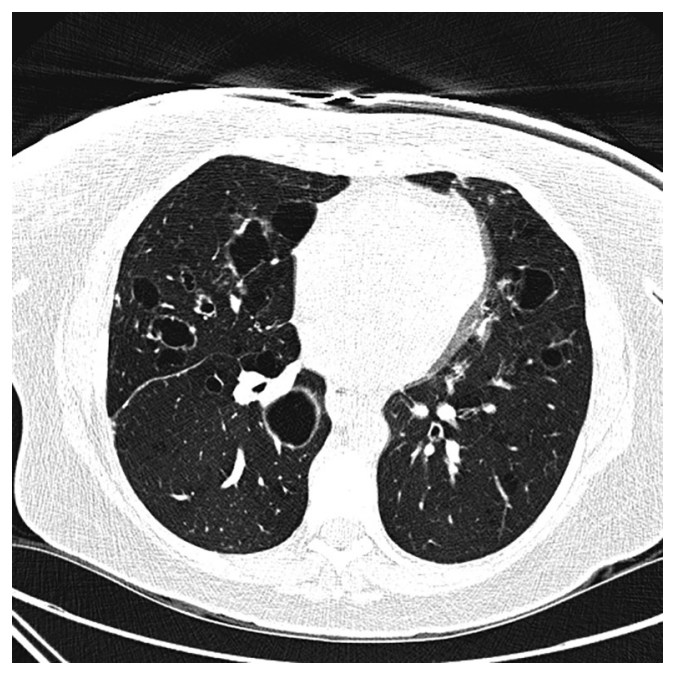

Figure 4 Thick-walled cysts in respiratory papillomatosis. 


\section{Self-assessment questions}

1. Which of the following is not a defining feature of a lung cyst?
a. Round circumscribed space
b. Surrounded by an epithelial wall
c. Thick walled (>4 mm)

2. Which of the following causes of cystic lung disease is not associated with an underlying genetic mutation?
a. PLCH
b. LAM (TSC-associated and sporadic)
c. BHD and other folliculin deficiencies

3. Which of the following is not an extrapulmonary manifestation of LAM?
a. Dilated cardiomyopathy
b. Renal AMLs
c. Chylous effusions (pleural or ascites)

\section{Infectious disorders}

Infectious agents are a well-documented cause of lung cysts, usually accompanying a history of either pneumonia or a systemic infection. Severe pneumonia has been associated with the development of pneumatoceles, particularly after staphylococcal infection in children, and in adults after mechanical ventilation [1-4]. Strictly defined, these are not cysts, but differentiating between them and cysts may be difficult, particularly if no prior chest imaging is available. Unlike other causes of cystic lung disease, radiological changes are reversible with treatment.

$P$. jirovecii is an opportunistic yeast-like fungal infection that most commonly affects immunocompromised patients, particularly those with HIV. Although formerly common, modern treatment for HIV and prophylaxis has made this rare [33].

Recurrent respiratory papillomatosis is caused by the human papillomavirus, particularly types 6 and 11 [34]. The radiographic features are of multiple solid or cystic nodules predominantly in the lower lobes, and sometimes resulting obstructive bronchiectasis and secondary infection [34] (figure 4). It can be treated with cidofovir [35].

\section{Conclusions}

The advent of modern diagnostic and laboratory techniques (including genetic, immunological, laboratory and molecular) has resulted in a rapidly improving understanding of the spectrum and genesis of cystic lung diseases. Far from being localised to the lung, these disorders are now recognised as being part of a range of systemic disorders. Learning about these diseases has challenged conventional views about the borderline between benign and malignant diseases, and enhanced our understanding of the causes of lung disorders. Environmental factors are currently under-recognised but undoubtedly important. No doubt, forthcoming decades will improve our understanding in this area and allow us to better evaluate the "whole person", account for individual factors in our patients. This should enable not only personalised treatment but also better prevention of these rare but important disorders.

\section{Key points}

- Cysts are a common incidental finding on computed tomography imaging of the lungs but can be missed unless high-resolution cuts have been performed. Diffuse cystic lung disease involves multiple cysts in more than one lung lobe. There is a wide differential diagnosis that includes orphan lung disorders, malignancies, interstitial lung diseases, infections and emerging disorders. Patients with multiple lung cysts are often diagnosed on the basis of radiological appearances alone and given a diagnostic label before full investigations are available; significant anxiety can result. Thus, patients may benefit from early referral to an expert centre.

- It is important to be able to distinguish a cyst from other pathological conditions, particularly emphysema, bullae, pneumatoceles, lung cavities and honeycomb lung.

- The differential diagnosis of cystic lung disease includes a broad range of genetic and acquired disorders, with classification based on cause. Occasionally, a definitive diagnosis cannot be made even with a lung biopsy. Lymphangioleiomyomatosis and folliculin deficiencies (e.g. Birt-Hogg-Dubé syndrome) are the most frequent causes, while inflammatory disorders, malignancies, infections, and occupational and environmental exposures are also implicated. If an underlying cause cannot be identified, a label of "cryptic-cause cystic lung disease" is used, acknowledging that this may be updated in the future.

- We are still learning about the pathophysiology and range of causes of multiple cystic lung diseases. Understanding in this area is rapidly evolving, allowing these diseases to become increasingly treatable. Early diagnosis is likely to improve prognosis. Investigation of cystic lung diseases needs to be thorough and painstaking, the respiratory equivalent of a detective's approach. 


\section{Affiliations}

Samantha Ennis', Elizabeth J. Silverstone², Deborah H. Yates'

'Dept of Thoracic Medicine, St Vincent's Hospital, Darlinghurst, Australia. .2Dept of Medical Imaging, St Vincent's Hospital, Darlinghurst, Australia.

\section{Suggested answers}

1. c.

2. a.

3. a.

\section{Conflict of interest}

None declared

\section{References}

1. Gupta N, Vassallo R, Wikenheiser-Brokamp KA, et al. Diffuse cystic lung disease. Parts 1 \& 2. Am J Respir Crit Care Med 2015; 191: 1354-1366.

2. Francisco FAF, Souza AS, Jr., Zanetti G, et al. Multiple cystic lung disease. Eur Respir Rev 2015; 24: 552-564.

3. Hansell DM, Bankier AA, MacMahon $\mathrm{H}$, et al. Fleischner Society: glossary of terms for thoracic imaging. Radiology 2008; 246: 697-722.

4. Araki T, Nishino M, Gao W, et al. Pulmonary cysts identified on chest CT: are they part of aging change or of clinical significance? Thorax 2015; 70: 1156-1162.

5. Cantin L, Bankier AA, Eisenberg RL. Multiple cystlike lung lesions in the adult. AJR Am J Roentgenol 2010; 194:W1-W11.

6. Cordier J-F, Johnson SR. Multiple cystic lung diseases. In: Cordier J-F, ed. Orphan Lung Diseases (ERS Monograph). Sheffield, European Respiratory Society, 2011; pp. 46-83.

7. Raoof S, Bondalapati P, Vydyula R, et al. Cystic lung diseases: algorithmic approach. Chest 2016; 150: 945-965.

8. Harari S, Torre O, Cassandro R, et al. The changing face of a rare disease: lymphangioleiomyomatosis. Eur Respir J 2015; 46: 1471-1485

9. Johnson SR, Cordier JF, Lazor R, et al. European Respiratory Society guidelines for the diagnosis and management of lymphangioleiomyomatosis. Eur Respir J 2010; 35: 14-26.

10. Gupta N, Finlay GA, KotloffRM, et al. Lymphangioleiomyomatosis Diagnosis and Management: High Resolution Chest Computed Tomography, Transbronchial Lung Biopsy and Pleural Disease Management. An Official Merican Thoracic Society/Japanese Respiratory Society Clinical Practice Guideline. Am J Respir Crit Care Med 2017; 196: 1337-1348.

11. Girschikofsky M, Arico M, Castillo D, et al. Management of adult patients with Langerhans cell histiocytosis: recommendations from an expert panel on behalf of Euro-Histio-Net. Orphanet J Rare Dis 2013; 8: 72.

12. Gupta N, Lee HS, Young LR, et al. Analysis of the MILES cohort reveals determinants of disease progression and treatment response in lymphangioleiomyomatosis. Eur Respir J 2019; 53: 1802066

13. Yates DH. mTOR treatment in lymphangioleiomyomatosis: the role of everolimus. Expert Rev Respir Med 2016; 10: 249-260.

14. Gupta N, Seyama K, McCormack FX. Pulmonary manifestations of Birt-Hogg-Dubé syndrome. Fam Cancer 2013; 12: 387-396.

15. Menko FH, van Steensel MA, Giraud S, et al. Birt-Hogg-Dubé syndrome: diagnosis and management. Lancet Oncol 2009; 10: 1199-1206.

16. Tobino K, Hirai T, Johkoh T, et al. Differentiation between Birt-Hogg-Dubé syndrome and lymphangioleiomyomatosis: quantitative analysis of pulmonary cysts on computed tomography of the chest in 66 females. Eur J Radiol 2012; 81: $1340-1346$.

17. Tschopp J-M, Bintcliffe O, Canalis E, et al. ERS task force statement: diagnosis and treatment of primary spontaneous pneumothorax. Eur RespirJ 2015; 46: 321-335.

18. Swerdlow SH, Campo E, Harris NL, et al. WHO classification of tumours of haematopoietic and lymphoid tissues. In:Weiss
LM, Jaffe R, Facchetti F, eds. WHO classification of Tumours of Haematopoietic and Lymphoid Tissues. Lyon, IARC, 2017; pp. 470-472.

19. Emile JF, Abla O, Fraitag $S$, et al. Revised classification of histiocytoses and neoplasms of the macrophage-dendritic cell lineages. Chest; 149: 1223-33.

20. Koyabaski M, Tojo A. Langerhans cell histiocytosis in adults: advances in pathophysiology and treatment. Cancer Sci 2018; 109: 3707-3713.

21. Vassallo R, Ryu JH, Schroeder DR, et al. Clinical outcomes of pulmonary Langerhans'-cell histiocytosis in adults. $N$ Engl J Med 2002; 346: 484-490.

22. Tazi A, de Margerie C, Naccache JM, et al. The natural history of adult pulmonary Langerhans cell histiocytosis: a prospective multicentre study. Orphanet J Rare Dis 2015; 10: 30.

23. Zamora AC, White DB, Sykes AM, et al. Amyloid-associated cystic lung disease. Chest 2016; 149: 1223-1233.

24. Gillmore JD, Hawkins PN. Amyloidosis and the respiratory tract. Thorax 1999; 54: 444-451.

25. Colombat M, Stern M, Groussard O, et al. Pulmonary cystic disorder related to light chain deposition disease. Am J Respir Crit Care Med 2006; 173: 777-780.

26. Mets OM, Schaefer-Prokop CM, de Jong PA. Cyst-related primary lung malignancies: an important and relatively unknown imaging appearance of (early) lung cancer. Eur Respir Rev 2018; 27: 180079

27. Barber E, Eapen A, Mehta R, et al. Benign metastasizing leiomyoma to the lung and spine: a case report and literature review. Case Rep Oncol 2019; 12: 218-223.

28. Swigris JJ, Berry GJ, Raffin TA, et al. Lymphoid interstitial pneumonia: a narrative review. Chest 2002; 122: 2150-2164.

29. Umehara $\mathrm{H}$, Okazaki $\mathrm{K}$, Masaki $Y$, et al. A novel clinical entity, IgG4-related disease (IgG4RD): general concept and details. Mod Rheumatol 2012; 22: 1-14.

30. Matsui S, Yamamoto H, Minamoto S, et al. Proposed diagnostic criteria for IgG4-related respiratory disease. Respir Investig 2016; 54: 130-132.

31. Chiarchiaro J, Tomsic LR, Strock S, et al. A case series describing common radiographic and pathologic patterns of hard metal pneumoconiosis. Respir Med Case Rep 2018; 25: $124-128$

32. Asaad A, Cao C, Rumbak M. Should hut lung be called domestically acquired particulate lung disease or domestically acquired pneumoconiosis? Respir Med Case Rep 2018; 23: 74-76.

33. Lu C-L, Hung C-C. Reversible cystic lesions of Pneumocystis jirovecii pneumonia. Am J Respir Crit Care Med 2012; 185: e7-e8.

34. Xiao $Y$, Wang J, Han D, et al. A case of the intrapulmonary spread of recurrent respiratory papillomatosis with malignant transformation. Am J Med Sci 2015; 350: 55-57.

35. Fortes HR, von Ranke FM, Escuissato DL, et al. Recurrent respiratory papillomatosis: a state-of-the-art review. Respir Med 2017; 126: 116-121. 